\title{
The Discovery of Red Low Surface Brightness Galaxies
}

\author{
Karen O'Neil \\ Arecibo Observatory, PO Box 995, Arecibo, PR 00613 \\ koneil@naic.edu
}

\begin{abstract}
We have performed a digital survey for Low Surface Brightness (LSB) galaxies in the spiral-rich Cancer and Pegasus clusters as well as the low density regime defined by the Great Wall. A total of 127 galaxies were found with $\mu_{B}(0)>22.0 \mathrm{mag} \operatorname{arcsec}^{-2}, 119$ of which were previously unidentified.

Consistent with other surveys, we find a significant number of galaxies with $\mu_{B}(0)>23.0 \mathrm{mag} \operatorname{arcsec}^{-2}$ which suggests that the space density of galaxies as a function of $\mu_{B}(0)$ is not strongly peaked. To more rigorously test this hypothesis we compare the actual surface brightness distribution from our survey with that from two different types of Monte-Carlo based sky images and show that it is not possible to distinguish between the flat distribution and the Gaussian one as the proper description of the underlying surface brightness distribution for this survey beyond 24.0 mag $\operatorname{arcsec}^{-2}$.

The colors of the survey galaxies range continuously from very blue to the first discovery of very red LSB galaxies. It also includes a group of old galaxies which show evidence for recent star formation. This continuous range of colors clearly shows that LSB galaxies at the present epoch define a wide range of evolutionary states.
\end{abstract}

\section{The Data}

All the data for this survey was taken on the University of Texas McDonald Observatory 0.8m telescope from 1993 - 1996 using a Loral-Fairchild 2048x2048 pixel CCD camera (1.32" / pixel resolution). Approximately fifty-four 45'x45' fields were imaged during that time, resulting in a total of 27 degrees $^{2}$ of sky being imaged. The fields covered both the Pegasus and Cancer sky regions as well as the area around various (known) galaxies distributed in the Great Wall (Geller \& Huchra 1989; Dell Antonio, Geller, \& Bothun 1996). Six to eight flat field images were taken each night for each filter and were (median) combined to obtain the flat field images for that night's data. The typical seeing disk was 1.5-2.0" during these observations, though we emphasize that in conditions of relatively good seeing the data are undersampled.

When conditions were photometric magnitudes and colors, corrected for atmospheric extinction, were obtained from both standard stars (Landolt 1973,1983) and by using the brighter galaxies in Bothun et al (1985) and Dell'Antonio, Geller, \& Bothun (1996) as photometric standards. The precision of these pho- 
$114 i 1$

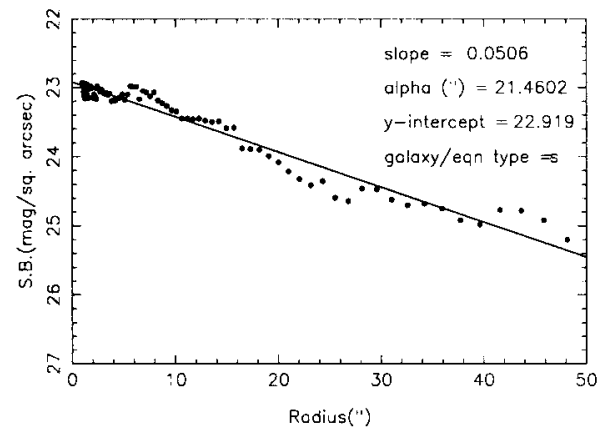

$\mathrm{v} 114$

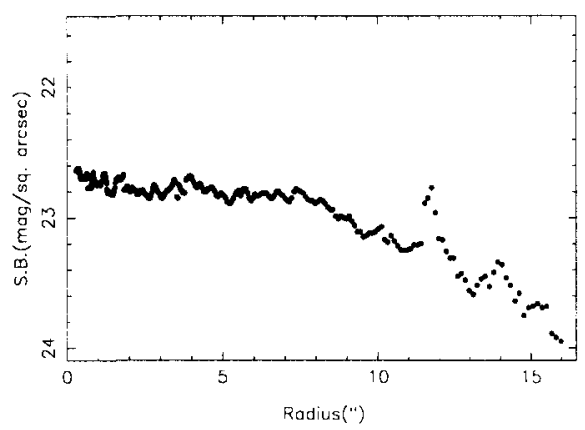

Figure 1. Surface brightness profiles of V1L4. The profile on the left (a) is from the Las Campanas 1.5-m telescope (B band), while on the right the profile (b) is from a HST WFPC-2 F814W (I band) image.

tometric standards is the lowest in the I-band filter and, in general, the signal-tonoise of the galaxies are also lowest in this filter (due to the increased noise and brightness in the sky background at I-band wavelengths). Typical precision for the I band photometric standards is $0.04 \mathrm{mag}$, while the secondary calibrators are good to $0.03 \mathrm{mag}$. Typically, three images were taken through both the B (exp. time 600s) and U (exp. time 900s) filters, and one was taken in V (300s) and I (120s). Multiple images were averaged to eliminate extraneous noise.

The average sky brightnesses through the $\mathrm{B}$ and I filters were $\langle\mathrm{B}\rangle=21.8 \pm$ $0.3 \mathrm{mag} \operatorname{arcsec}^{-2}$ and $\langle\mathrm{I}\rangle=18.5 \pm 0.5 \mathrm{mag} \operatorname{arcsec}^{-2}$. The sky was often brighter than would have been ideal due to the presence of thin cirrus clouds during many of our observations (hence requiring the use of previously observed galaxies in the field to act as photometric standards). On average we were able to detect LSB galaxies down to $\mu_{B}(0)=25.5 \mathrm{mag} \operatorname{arcsec}^{-2}$, or $3 \%$ of the sky background. Surface brightness profiles could accurately (error $\leq 0.25 \mathrm{mag} \mathrm{arcsec}^{-2}$ ) be measured to $27.5 \mu_{B}$, or $0.5 \%$ of the sky background. Since images of LSB galaxies are difficult to print, we refer the reader to http://guernsey.uoregon.edu/ karen where all the digital data is reproduced.

Further details about the data reduction, zeropoint determination, and galaxy identification can be found in O'Neil, Bothun, \& Cornell (1997) and O'Neil, et al (1997) (OBC and OBSCI, respectively).

\section{Central Surface Brightness}

Once galaxies were identified, the galaxy's peak intensity was found and ellipses were fit around that point to obtain the intensity in each annulus using the GASP software package (Cawson 1983). In the cases where no obvious peak intensity existed in the galaxy (the clumpier LSB galaxies) the physical center, estimated by centroiding with respect to the outer isophotes, of the galaxy was chosen. Because of the coarse pixel size of many of the galaxies in this survey, our accuracy in locating galactic centers was only good to within 2". Surface brightness profiles and other data cannot be trusted inside that boundary, which 
also corresponds well with the typical size of the seeing disk. The average skysubtracted intensity within each (annular) ellipse was found and calibrated with the determined photometric zeropoint.

Surface brightness profiles were then plotted against the major axis (in arcsec) and a best fit line was found. The majority of the galaxies in this survey $(80 \%$, or 102 galaxies) being well fit by an exponential profile:

$$
\mu(\mathrm{r})=\mu(0)+\left(\frac{1.086}{\alpha}\right) \mathrm{r}
$$

where $\mu(0)$ is the central surface brightness in (mag $\left.\operatorname{arcsec}^{-2}\right)$ and $\alpha$ is the scale length in arcsec. In $17 \%$ of the galaxies, though, the exponential fit was clearly not representative of the light profile. In these cases we found that the surface brightness profiles were much better fit (in the reduced $\chi^{2}$ sense) with King's (1965) model of star clusters, approximated by:

$$
\mu(\mathrm{r})=\mu(0)+2.5 \log \left(1+\left(\frac{\mathrm{r}}{\mathrm{R}_{\mathrm{E}}}\right)^{\mathrm{n}}\right)
$$

where $\mu(0)$ again is the central surface brightness in (mag $\left.\operatorname{arcsec}^{-2}\right), R_{\mathrm{E}}$ is the core radius, and $\mathrm{n}$ is a free parameter. Finally, it should be noted that $3 \%$ of the galaxies (4) could not be fit by any curve, while none of the galaxies were fit by a $\mathrm{r}^{1 / 4}$-type (elliptical) profile.

All that will be noted herein about those galaxies best fit by a King profile is simply that the galaxies' surface brightness profiles are similar to those found in high resolution imaging of known LSB galaxy cores. Figure 1(a) shows a typical exponential profile of a nearby LSB galaxy (V1L4) taken with the Las Campanas 2.5-m telescope (Impey, Bothun, \& Malin 1988). To its right, Figure 1(b) shows the surface brightness profile of the same galaxy as imaged with the HST Wide Field Planetary Camera-2 (0.0996" / pixel resolution) (O'Neil, Bothun, \& Impey 1998). As all four LSB galaxies imaged by O'Neil, Bothun, \& Impey (1998) show both an overall exponential profile and this flat inner core (see Figure 1 b), it seems likely that the presence of a flat inner core is not uncommon in LSB galaxies. This leads to the hypothesis that the galaxies we categorized as 'king profile' are galaxies whose size is large enough and surface brightness profile faint enough that only the inner core of the galaxies could readily be discerned.

The central surface brightness distribution of the exponential profile (disklike) galaxies is given in Figure 2, which shows a flat profile from $\mu_{B}(0)=22.0$ mag arcsec -2 through $24.0 \mathrm{mag} \operatorname{arcsec}^{-2}$ and a sharp drop-off on either side. The drop-off at $\mu_{B}(0)=22.0 \mathrm{mag} \operatorname{arcsec}^{-2}$ is purely a factor of our selection criteria and should be ignored as such. The drop-off at $24.0 \mathrm{mag} \operatorname{arcsec}^{-2}$, though, is worthy of further investigation as it occurs well before the detection threshold of $\mu_{B}(0) \sim 26.5 \mathrm{mag} \operatorname{arcsec}^{-2}$.

Three different tests were run in an attempt to determine whether the central surface brightness drop-off at $24.0 \mathrm{mag} \mathrm{arcsec}-2$ is a real effect (possibly due to the dense cluster environment) or simply further evidence of our selection biases. The first two tests were based off the assumed knowledge of the cluster 


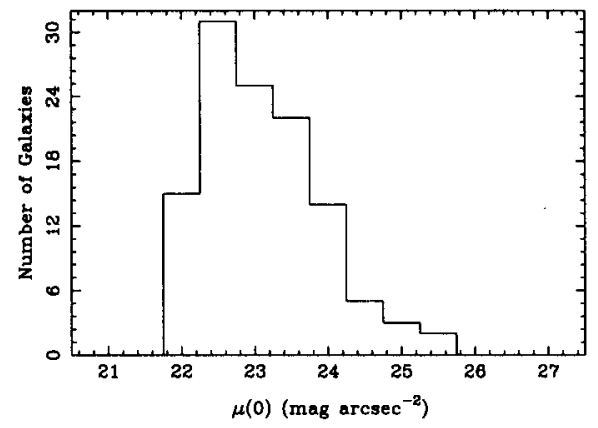

Figure 2. Distribution of $\mu_{B}(0)$ for the galaxies found in this survey.

densities surrounding each galaxy. ${ }^{1}$ The first test utilized the 2-dimensional galaxy density in each field to look for trends between this density and the average central surface brightness and scale length in the fields. A definite trend toward brighter average central surface brightness and smaller average scale lengths with increased density was found, indicating cluster densities do affect the central surface brightness and scale lengths of galaxies. The second test simply separated the galaxies into three groups based on their cluster (or lack thereof) identity and looked for trends between these three groups (Pegasus, Cancer, and Great Wall galaxies). In this case, no difference between the three groups' central surface brightness or scale length distribution was found. Thus, the second test led to the conclusion that the cluster environment plays no role in determining a galaxy's morphology, directly in contradiction with the results of the first test.

Finally, in an attempt to understand whether or not we would be able to detect a difference in the underlying central surface brightness distribution were it to (a) fall off as a Gaussian after $\mu_{B}(0)=23.0 \mathrm{mag} \operatorname{arcsec}^{-2}$ or (b) be completely flat from $22.0 \mathrm{mag} \operatorname{arcsec}^{-2}$ through $28.0 \mathrm{mag} \mathrm{arcsec}^{-2}$, a series of Monte Carlo simulations were run. The simulations were based off these two different underlying surface brightness distributions and continued the same sky brightness, noise, number of stars, etc. as the original Texas data (see OBSCI for details). Twelve fields of each distribution were created and analyzed, with no detectable difference between the galaxies detected with Model A (the Gaussian distribution), the galaxies detected with Model B (the flat distribution), and the galaxies detected from the Texas survey. The final result, then, is that there is no way to determine whether the observed central surface brightness cut-off at $\mu_{B}(0)=24.0 \mathrm{mag} \operatorname{arcsec}^{-2}$ is due to the effect a cluster environment has

\footnotetext{
${ }^{1}$ It should be noted for both these tests that the redshifts of the individual galaxies were not known. Instead, an assumed distance was given to the galaxies based on the distances to other known galaxies within each field. Redshifts were estimated due to the unfortunate timing of the galaxies' discovery with the closing of the Arecibo 100-m telescope for refurbishment. As the refurbishment is now complete redshifts of these galaxies have been obtained and re-analysis of the data is underway. See O'Neil, et al (1999) for further details.
} 

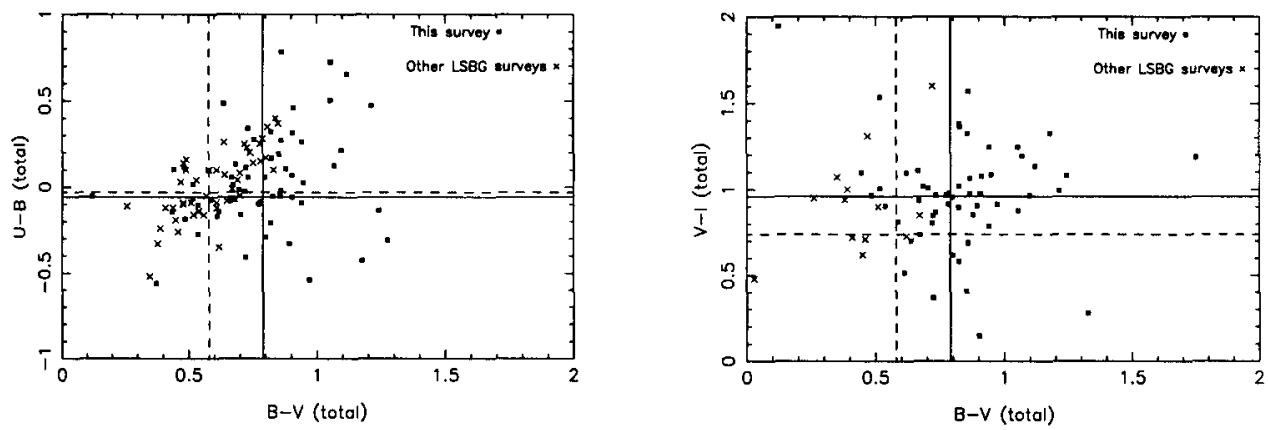

Figure 3. U-B, B-V, and V-I colors both for all the galaxies found in this survey (squares) as well as for numerous other LSB galaxy surveys (de Blok, van der Hulst, \& Bothun 1995; Sprayberry, et al 1995; McGaugh 1992; Bothun, et al 1991; Impey, Bothun, \& Malin 1988; Caldwell \& Bothun 1987; Romanishin, Strom, \& Strom 1983)

on galaxy morphologies, or due to the same selection effects which prevented significant detection of LSB galaxies for many decades.

\section{Color}

The use of multiple filters (Johnson/Kron-Cousins U,B, V, \& I) for the sky images in this survey allowed for the determination of broadband colors for most of the galaxies discovered. Figure 3 shows the color distribution for both the galaxies discussed herein as well as for most other LSB galaxies with known colors. In an attempt to describe these colors, the galaxies will be separated into three categories - the very blue, the very red, and everything else.

\subsection{The Very Blue}

The galaxies in this category are those with colors of $\mathrm{U}-\mathrm{B}<-0.2, \mathrm{~B}-\mathrm{V}<0.6$, $\mathrm{V}-\mathrm{I}<1.0$. The existence of very blue LSB galaxies has been previously documented (i.e. McGaugh \& Bothun 1994; de Blok, van der Hulst, \& Bothun 1995; Impey, et al 1996) and is important primarily because of the restrictions they put on the star formation history of LSB galaxies. The simplistic explanation - that LSB galaxies are merely faded remnants of HSB galaxies - is quickly eliminated by the existence of these very blue LSB galaxies. The average colors of $\mathrm{E} 0 / \mathrm{S} 0$ galaxies (good examples of old stellar populations) are $\mathrm{U}-\mathrm{B}=0.54$, $\mathrm{B}-\mathrm{V}=0.96$ (Tinsley 1978), while late type spiral galaxies have colors similar to these very blue LSB galaxies. Sc or later spiral galaxies with active star formation typically have colors in the range $0.35 \leq \mathrm{B}-\mathrm{V} \leq 0.65,-0.2 \leq \mathrm{U}-\mathrm{B} \leq-0.4$ (Huchra 1977; Bothun 1982), with mean values $\langle\mathrm{B}-\overline{\mathrm{V}}\rangle \approx 0.50,\langle\mathrm{U}-\mathrm{B}\rangle \approx-0.20$ (Bothun 1982) and $\langle\mathrm{V}-\mathrm{I}\rangle \approx 1.0$ (Han 1992). These colors encompass most of the very blue galaxies in our survey, and come close to matching the LSB galaxy colors found by McGaugh \& Bothun (1994) (median colors of $\langle\mathrm{B}-\mathrm{V}\rangle=0.44$, $\langle\mathrm{U}-\mathrm{B}\rangle=-0.17$, and $\langle\mathrm{V}-\mathrm{I}\rangle=0.89)$ and Romanishin, Strom, \& Strom 
(1983) (average colors of $\langle\mathrm{B}-\mathrm{V}\rangle=0.43 \pm 0.04,\langle\mathrm{~V}-\mathrm{R}\rangle=0.60 \pm 0.02$ ). Of course it should be noted that LSB disk galaxies, on average, have factors of ten lower star formation rates (SFRs) than Sc galaxies (i.e. McGaugh \& Bothun 1994; McGaugh \& de Blok 1997).

The issue here is how to best explain these very blue colors in galaxies with very low SFRs. One explanation for the very blue LSB galaxies could be their low metallicity. McGaugh (1994) has shown that LSB galaxies typically have metallicities approximately $1 / 3$ the solar metallicity. This could lead to a bluing of the galaxies' colors. A comparison with the colors of globular clusters $(\langle\mathrm{U}-\mathrm{B}\rangle \approx 0.1,\langle\mathrm{~B}-\mathrm{V}\rangle \approx 0.65,\langle\mathrm{~V}-\mathrm{I}\rangle \approx 0.95$ (Reed 1985)), however, shows that these very blue LSB galaxies are actually bluer than very metal poor globular clusters. Thus, although low metallicity may play a role in the color of very blue LSB galaxies, it can only account for part of the reason (see McGaugh \& Bothun 1994; de Blok, van der Hulst, \& Bothun 1995). For these and many other reasons (i.e. O'Neil, Bothun, \& Schombert 1998; Bothun, Impey, \& McGaugh 1997; de Blok, van der Hulst, \& Bothun 1995; McGaugh 1992), the simplest surviving theory to explain the very blue LSB galaxies is that the galaxies have a young mean age.

\subsection{The Very Red}

A new and significant discovery in this survey are very red LSB galaxies. As seen in Figure 3, before this study few LSB galaxies found had $U-B>0.3$, $\mathrm{B}-\mathrm{V}>0.8$, and no previous survey had found LSB galaxies with $\mathrm{U}-\mathrm{B}>0.4$ and $\mathrm{B}-\mathrm{V}>0.9$. Detailed studies (OBSCI) show these red colors are unlikely to be the result of photometric errors and/or the presence of strong bulges. The red colors are also unlikely to be the manifestation of large internal reddening, as there is little evidence of dust in LSB disks in general (i.e. Schombert et al 1992; McGaugh 1994; OBSCI). This may reflect both the relatively unevolved state of LSB disks and/or a deficiency of massive stars born in each, sporadic star formation event.

This lack of detected red LSB galaxies has been a concern in the past (i.e. McGaugh \& Bothun 1994) because without them it appears LSB galaxies have colors equivalent only to Sc and later galaxies and do not cover the full spectrum of HSB galaxy colors - that is, it appears no faded LSB galaxies exist at the present epoch. Since at some future epoch when star formation ceases, all galaxies will fade into LSB objects, the lack of this process occurring now, given the low fractional gas contents of many galaxies, is a bit disconcerting. Indeed, McGaugh (1994) has argued that the photographic selection process has prevented red LSBs disks from being detected previously so perhaps the CCD selection criteria, defined by simultaneous detections in the B, and V filters, has effectively corrected for this selection effect. Possibly, as argued below, LSB disk galaxy evolution is accelerated in clusters and hence clusters contain more red LSB disks than blue LSB disks, although in general this is not the case for HSB disks in spiral-rich clusters (e.g. Bothun et al 1982) nor really for dwarf galaxies in the Fornax cluster (e. g. Evans, Davis, \& Phillips 1990; Bothun et al 1991).

To test if the selection through multiple filters influenced our finding of red LSB galaxies, we compared the $(\mathrm{B}-\mathrm{V})$ color distribution for those galaxies within $1 \sigma$ of the average $\left\langle\mu_{B}(0)\right\rangle$ against the the $(\mathrm{B}-\mathrm{V})$ colors of the galaxies 
within $1 \sigma$ of $\left\langle\mu_{V}(0)\right\rangle$. Both distributions had $\langle B-V\rangle=0.72 \pm 0.1$, and a mean value of $B-V=0.69$. Thus, had images only been available in one of the two filters $(\mathrm{B}$ or $\mathrm{V})$, the same overall color distribution, including the very red LSB galaxies, would still have been found.

The average colors of $\mathrm{E} 0 / \mathrm{S} 0$ galaxies are $\langle\mathrm{U}-\mathrm{B}\rangle=0.54,\langle\mathrm{~B}-\mathrm{V}\rangle=0.96$ (Tinsley 1978). The galaxies in the upper right corner of Figure 3(a) \& (b) include colors from $0.8 \leq \mathrm{B}-\mathrm{V} \leq 1.2,0.4 \leq \mathrm{U}-\mathrm{B} \leq 0.8$, and $1.0 \leq \mathrm{V}-\mathrm{I} \leq 1.7$, showing that the colors of the very red LSB galaxies in our survey are similar to the colors of some of the oldest galaxies. Larson and Tinsley (1978) approximate these colors with their model of a galaxy which formed stars for only the first $10^{7}$ years, and has been quiescent for it's remaining 20 Gyrs. (For this model they obtain $\mathrm{U}-\mathrm{B}=0.74, \mathrm{~B}-\mathrm{V}=1.02)$. It is quite likely, then, that these galaxies are also the result of a bursting SFR. Unlike their blue counterparts, though, these galaxies underwent starburst early in their existence, consuming most of the galaxies' gas and leaving them with only an old stellar population. In fact, this is the expected route to low disk galaxy surface brightness but until now, it had not been observationally detected.

\subsection{The Rest}

Naturally, the rest of the galaxies in our survey fall somewhere between the last two extremes - neither consisting of only a young, blue population nor containing only old, red stars. These galaxies fill in the gap between the very blue LSB galaxies and the very red LSB galaxies, showing that we are not merely seeing two distinct galaxy groups - a group of very young, LSB galaxies and a group of very old, faded galaxies - but instead that LSB galaxies cover the entire HSB color spectrum.

One significant group of galaxies have colors $\mathrm{U}-\mathrm{B}<-0.2, \mathrm{~B}-\mathrm{V}>0.8$, and $\mathrm{V}-\mathrm{I}<1.0$, corresponding to primarily young to 'middle-aged' (A0-G8) stars and lack a large population of very old, red stars. The colors of these galaxies are reproduced by Guiderdoni \& Rocca-Volmerange $(1987,1988)$ (GR from now on) for young $\mathrm{Sc} / \mathrm{Sd}$ galaxies. $\mathrm{GR}$ obtains the colors $\mathrm{U}-\mathrm{B}=-0.18--0.27$, $\mathrm{B}-\mathrm{V}=0.75-0.88$, and $\mathrm{V}-\mathrm{I}=0.91-1.00$ for $\mathrm{Sc} / \mathrm{Sd}$ galaxies $11.5-11.8$ Gyrs old (using GR's values of $h_{0}=0.5$ and $\Omega=0.1$ ). GR assumed the galaxies would have a heavy element fraction of $Z=0.02$, an unrealistic assumption for most LSB galaxies. McGaugh (1994) gives LSB galaxies a heavy element fraction of $\mathrm{Z}<30 \% Z_{\odot}$, or $\mathrm{Z}<0.01$. Reducing the metallicity of the galaxies in GR would primarily reduce their values for $\mathrm{V}-\mathrm{I}$, which follows the trend of the galaxies in our survey quite nicely. As the actual age of these galaxies is presumably similar to the age of all the other galaxies in the various clusters (i.e. Pegasus, Cancer), linking them with galaxies only $11.6 \mathrm{Gyr}$ old merely indicates they have a lower overall SFR than their red counterparts.

This group of galaxies is well represented by galaxies which have already experienced their primary phase of starburst but have not completely left their star formation era. These galaxies most likely represent the next evolutionary phase for the very blue LSB galaxies mentioned earlier.

The other interesting galaxy group consists of galaxies whose $\mathrm{B}-\mathrm{V}$ and $\mathrm{V}-\mathrm{I}$ colors indicate a large, old stellar population, yet whose U-B colors expose a population of young stars. That is, they appear to be old galaxies currently 
undergoing a small yet significant burst of star formation. In all cases the very red V-I or B-I colors can only be obtained by a significant population of K0 and later stellar types, yet the $\mathrm{U}-\mathrm{B}$ colors in this group are similar to a Sc or earlier galaxy (Huchra 1977).

Results from the models of O'Neil, Bothun, \& Schombert (1998) shows that if $15 \%$ of a galaxy undergoes a starburst, the galaxy's U-B color will change by $-0.6 \mathrm{mag}$ while it's $\mathrm{B}-\mathrm{V}$ and $\mathrm{V}-\mathrm{I}$ colors change by only -0.3 and $-0.15 \mathrm{mag}$, respectively. If the starburst is evenly distributed throughout the galaxy the central surface brightness will increase by roughly $1 \mathrm{mag} \mathrm{arcsec}^{-2}$. Realistically, though, it is likely tidal interactions will result in local compressions in the LSB galaxy's gas (Mihos, McGaugh, \& de Blok 1997). In this case, the color changes would occur in outer annuli and the galaxy's central surface brightness would show a smaller increase. Note that rotation curve data obtained by de Blok, McGaugh, \& van der Hulst (1996) indicates that LSB disks are dark matter dominated at all radii and hence are likely to be quite stable to tidal disruption so repeated encounters in the cluster environment are possible. In addition, most LSB disks are in an environment that would promote only weak tidal interactions (Bothun et al 1993).

\section{Summary}

Our survey resulted in the detection of 127 galaxies in the local universe with $\mu_{B}(0)>22.0 \mathrm{mag} \operatorname{arcsec}^{-2}, 119$ of which were previously undetected. The central surface brightness distribution of the discovered galaxies is flat from $22.0 \mathrm{~B}$ mag $\operatorname{arcsec}^{-2}$ (the artificial survey cut-off) through $24.0 \mathrm{mag} \operatorname{arcsec}^{-2}$, at which point there is a sharp drop-off. Through a series of Monte Carlo simulations, though, we've shown that there is no way of knowing whether this cut-off is real or due to selection effects.

Additionally, this survey found LSB galaxy colors covering the entire HSB galaxy color spectrum, including the first discovery of a population of very red LSB galaxies. All of the galaxy colors in this survey can be explained through a low overall star formation rate with sporadic bursts of star formation, and through modeling we determined that these bursts of star formation will not significantly increase the galaxies' central surface brightness.

Acknowledgments. Thanks to Greg Bothun, Mark Cornell \& Jim Schombert for help with this work. This work was done while at the University of Oregon.

\section{References}

Bothun, G.D., Impey, C., \& McGaugh, S. 1997 PASP 109, 745

Bothun, G., et al 1993, AJ, 106, 530

Bothun, G., et al 1991, ApJ, 376, 404

Bothun, G., et al 1985, ApJS, 57, 523

Bothun, G., et al 1982, AJ, 87, 725

Bothun, G. 1982, PASP, 94, 774

Caldwell, N. \& Bothun, G. 1987, AJ, 94, 1126 
Cawson, M. 1983, Ph.D. thesis, University of Cambridge

de Blok, W., McGaugh, S. \& van der Hulst, J. 1996, MNRAS, 283, 18

de Blok, W., van der Hulst, J., \& Bothun. G. 1995, MNRAS, 274, 235

Dell'Antonio, I., Geller, M., \& Bothun, G. 1996, AJ, 112, 1780

Evans, R., Davies, J., and Phillipps, S. 1990 MNRAS, 245, 164

Geller, M., \& Huchra, J. 1989, Sci, 246, 897

Guiderdoni, B. \& Rocca-Volmerange, B. 1988, A\&AS, 74, 185 (GR)

Guiderdoni, B. \& Rocca-Volmerange, B. 1987, A\&A, 186, 1 (GR)

Han, M. 1992, ApJS, 81, 35

Huchra, J. 1977, ApJS, 35, 171

Impey, C., Sprayberry, D., Irwin, M., \& Bothun, G., 1996, ApJS, 105, 209

Impey, C., Bothun, G., \& Malin, D. 1988, ApJ, 330, 634

King, I. 1965, AJ, 71, 64

Landolt, A. 1983, AJ, 88, 439

Landolt, A. 1973, AJ, 78, 959

Larson, R., \& Tinsley, B. 1978, AJ, 219, 46

McGaugh, S., de Blok, W. J. 1997, ApJ, 481, 689

McGaugh, S. 1994, ApJ, 426, 135

McGaugh, S. \& Bothun, G. 1994, AJ, 107, 530

McGaugh, S. 1992, Ph.D. thesis, University of Michigan

Mihos, C., McGaugh, S., \& de Blok, W. 1997, ApJ, preprint

O'Neil, et al 1999 in preparation

O'Neil, K., Bothun, G., \& Impey, C. 1998 submitted to AJ

O'Neil, K., Bothun, G., Schombert, J. 1998 AJ, preprint

O'Neil, K., et al 1998 AJ, 116, 657

O'Neil, K., et al 1997 AJ, 114, 2448 (OBSCI)

O'Neil, K., Bothun, G., \& Cornell, M. 1997 AJ, 113, 1212 (OBC)

Reed, B. 1985, PASP, 97120

Romanishin, W., Strom, K., \& Strom, S. 1983, ApJS, 53, 105

Schombert, J., Bothun, G., Schneider, S., \& McGaugh, S. 1992, AJ, 1031107

Sprayberry, D., Impey, C., Bothun, G., \& Irwin, M. 1995, AJ, 109, 558

Tinsley, B. 1978, ApJ, 222, 14 